\title{
STRUCTURAL AND MECHANICAL CHARACTERIZATION OF DEFORMED POLYMER USING CONFOCAL RAMAN MICROSCOPY AND DSC
}

\author{
Birgit Neitzel*, Florian Aschermayer, Milan Kracalik, Sabine Hild \\ Johannes Kepler University Linz, Institute of Polymer Science, Altenberger Straße 694040 Linz, Austria \\ * corresponding author: Birgit.Neitzel@jku.at
}

\begin{abstract}
Polymers have various interesting properties, which depend largely on their inner structure. One way to influence the macroscopic behaviour is the deformation of the polymer chains, which effects the change in microstructure. For analyzing the microstructure of non-deformed and deformed polymer materials, Raman spectroscopy as well as differential scanning calorimetry (DSC) were used. In the present study we compare the results for crystallinity measurements of deformed polymers using both methods in order to characterize the differences in micro-structure due to deformation. The study is ongoing, and we present the results of the first tests.
\end{abstract}

KEYWORDS: Raman spectroscopy, DSC, deformed polymers, crystallinity.

\section{INTRODUCTION}

Deformed polymers are used in a wide range of applications because of their advanced mechanical properties. Especially, materials with high strength gained increasing importance. In order to obtain high strength materials with tailored properties, the morphology can be controlled by varying process conditions. Nevertheless, there is still a lack of knowledge in the correlation between mechanical properties, processing conditions and polymer morphology. The microscopic structure is claimed as a crucial factor, which determines the mechanical properties. Furthermore, mechanical performance is influenced by the crystallinity and chain orientation of a polymer. Therefore, characterization of these parameters is important for developing new tailored polymeric materials.

Wide angle X-ray diffractometry or differential scanning calorimetry (DSC) are common methods to determine the overall crystallinity of polymers. Besides, also spectroscopic techniques such as Raman spectroscopy [1 4] have shown to be suitable techniques to observe process-induced changes in crystallinity [5] and chain orientation 3 .

Raman spectroscopy uses polarized laser light to scan the surface of a sample. When changing the polarization angle of the filter, the intensities of certain structure-related peaks in the Raman spectrum change as well, which allows conclusions on the polymer morphology. The degree of orientation can be determined from these peaks via a material specific orientation function $f_{c z}$, such as Equation (1) for PP [3]:

$$
f_{c z}=0.134 \cdot \frac{\sum I_{973}}{\sum I_{998}}-0.182
$$

It reveals if the polymer chains are randomly distributed $\left(f_{c z}=0\right)$, in deformation direction $\left(f_{c z}>0\right)$ or perpendicular to it $\left(f_{c z}<0\right)$.

As $f_{c z}$ depends on the polarization filter's orientation, the differences in multiple spectra must be considered when determining its value. Crystallinity $\alpha$ is supposed to be independent of the filter's orientation. Its equation sums up peaks of multiple spectra to determine its value.

$$
\alpha_{\text {Raman }}=\frac{\sum I_{809}}{\sum I_{809}+\sum I_{841}}
$$

Directed force is applied to the samples, this direction is designated MOD (machine orientation direction). For each sample two Raman spectra were recorded. The first with polarisation set to 0 degrees, emitting laser light perpendicular to the MOD. The second spectrum is taken with the laser aligned parallel (i.e. 90 degrees) to the direction of the applied force. We took 40000 measurements of one sample per MOD to see if MOD-dependent peaks in the spectra can be identified. The relative intensities were determined by integration of Raman bands. The crystallinity was then calculated via Equation (2) from the sum-spectra of both measurement series, parallel and perpendicular to the deformation direction.

The second method we used, was DSC. From DSC curves, first the enthalpy of the sample can be calculated and from these results the crystallinity as well. One goal of this study is to compare the crystallinity values, obtained from both methods, because each technique is best suited for different surface textures. If a sample has a very smooth surface, then confocal Raman microscopy can be used; for more irregular surfaces, measuring with DSC is more suitable. To characterize the polymer sample also on a macro-structure level, mechanical tests are ongoing. 
The mechanical investigation focuses on tensile testing. With this method, tensile strength and fracture behaviour can be analysed. Furthermore, hardness tests could give us important data to build up the comprehensive material overview of the polymer samples in micro- and macro structure scales.

\section{EXPERIMENTAL}

In this current study, Raman measurements were done with a confocal Raman microscope (Alpha300R, Witec GmbH, Ulm, Germany). For the measurements, polarized laser light of $532 \mathrm{~nm}$ and $16 \mathrm{~mW}$ power was used. A polarization direction of $0^{\circ}$ or $90^{\circ}$ was applied. The graphical analysis has been performed using an objective with 20x magnification. With this equipment and these settings, polypropylene (PP) samples with different degrees of deformation in one direction were analyzed. Furthermore, polyethylene terephthalate (PET) samples were investigated. The second method in this study was DSC using the instrument Perkin Elmer, DSC 8000, USA . Both, heating rate and cooling rate were set at $10 \mathrm{~K} / \mathrm{min}$. The $\mathrm{PP}$ samples were heated from $25^{\circ} \mathrm{C}$ to $250^{\circ} \mathrm{C}$, and the PET samples from $25^{\circ} \mathrm{C}$ to $300^{\circ} \mathrm{C}$. The crystallinity was calculated from the first heating curve, to detect the influence, caused by the deformation.

\section{Results And Discussion}

\subsection{Crystallinity Determined By Confocal Raman Microscopy}

In the case of PP, the peaks at $809 \mathrm{~cm}^{-1}$ and $841 \mathrm{~cm}^{-1}$ provide information about the crystallinity, whereas the peaks at $973 \mathrm{~cm}^{-1}$ and $998 \mathrm{~cm}^{-1}$ are important for determining the chain orientation. These peaks change their orientation depending on the polarization of the laser light. All results were calculated via integration of the peak areas (see Figure 1).

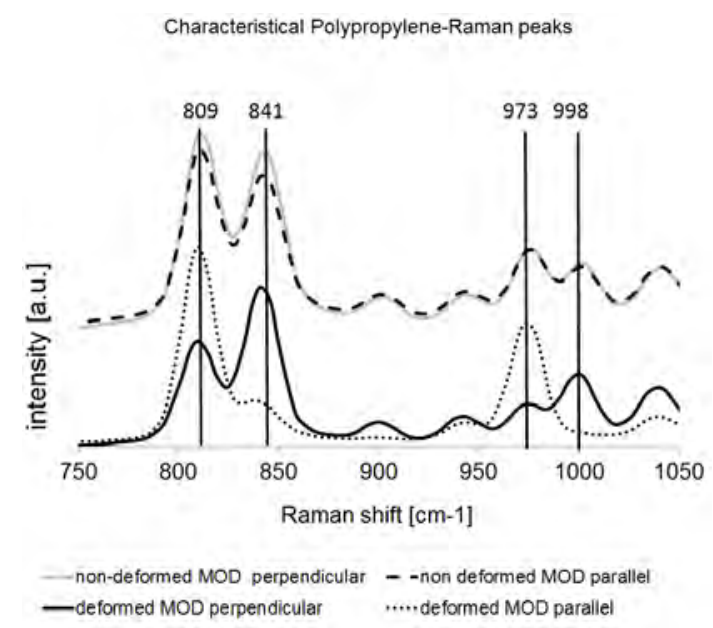

Figure 1. Material specific (PP) peaks for crystallinity and chain-orientation calculation.

In Figure 2 the crystallinity distribution of a nondeformed (Figure 2a), deformed PP (Figure 2b), and highly deformed PP (Figure 2c) can be seen. The deformed sample has a higher degree of crystallinity compared to the non-deformed sample. Thus, the random distribution of the polymer chains, in the nondeformed sample changed to a well-ordered structure in the highly deformed PP-sample.

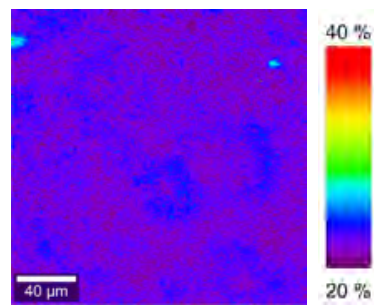

(A) . non-deformed PP

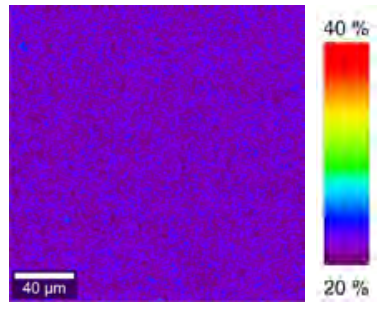

(B) . deformed PP

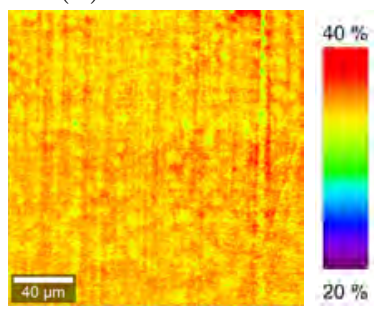

(C) . highly deformed PP

Figure 2. Crystallinity $\alpha$ calculated from Raman spectra.

Confocal Raman microscopy can be a powerful tool for structural analysis of further polymeric materials, but in this case it is necessary to find material specific structure relevant peaks. Two peaks could be interesting for the second investigated material, PET: $998 \mathrm{~cm}^{-1}$ and $1096 \mathrm{~cm}^{-1}$. 6] Furthermore, material specific formulas for crystallinity and orientation are required.

\subsection{Crystallinity Obtained by DSC}

A big advantage of differential DSC is that samples can be produced very easily and in the same way for every polymer. Also the crystallinity can be calculated with the same equation. So far, PET has been analysed as a second polymeric material. In Figure 3, the crystallinity, calculated from DSC measurements, can be seen.

These first measurements show, that crystallinity increases with high deformation. In a next step, these results will be compared to the crystallinities, determined via confocal Raman microscopy. Macrostructure will be characterized via tensile test. The aim is to find a relationship between the micro- and 


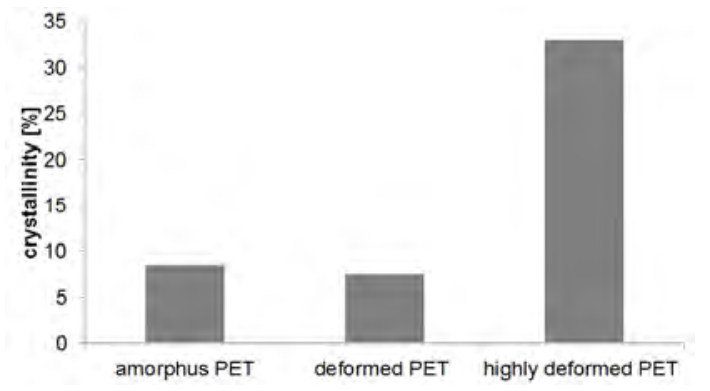

Figure 3. Crystallinity of PET samples, measured via DSC; (A) amorphous, (B) deformed and (C) highly deformed PET.

macro-structure behaviour of different polymeric materials.

\subsection{Comparison of Crystallinity Calculated by Confocal Raman Microscopy AND DSC}

Using both methods, DSC and Raman spectroscopy, it was found that the crystallinity $\alpha$ (Figure 4 ) increases with higher deformation, but they have different increase rates.

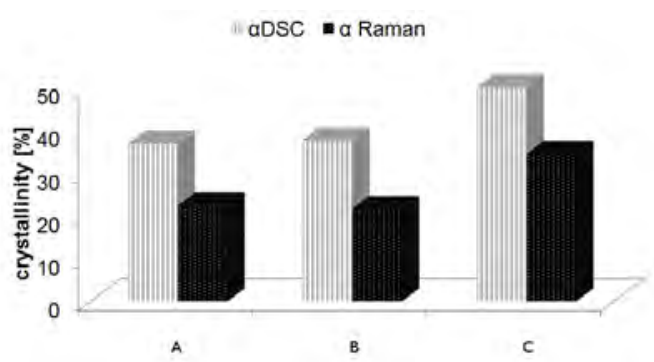

Figure 4. Crystallinity $\alpha$ calculated from Raman spectra and DSC measurements for $A$ non-deformed, $B$ deformed and $C$ highly deformed polypropylene (PP).

The highest crystallinity and best correlation between both measuring methods was observed in the most deformed polypropylene $C$. It was furthermore observed that deformation of PP causes changes in its microstructure.

\subsection{Orientation Distribution Obtained By Confocal Raman Microscopy}

Figure 5 shows that the orientation function for the polymer chains, determined via confocal Raman microscopy, increases with higher deformation. The polymer chains realign under stress by positioning themselves parallel to each other, resulting in a restructured polymer. This effect was also observed for the crystallinity, which also increases. $A$ denotes non-deformed PP without orientation, $B$ deformed polymer with weak orientation and $C$ highly deformed $\mathrm{PP}$ with a strong chain-orientation along the deformation direction, $0^{\circ}$ perpendicular, $90^{\circ}$ parallel to MOD.

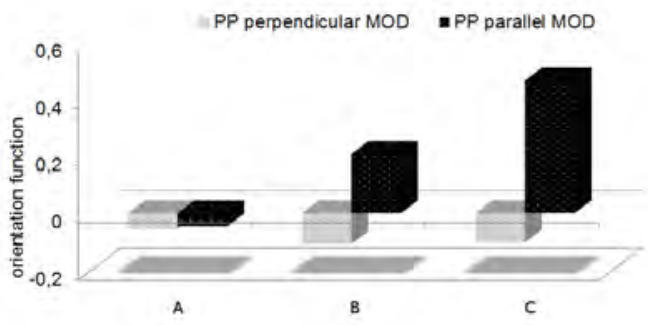

Figure 5. Orientation function calculated from Raman spectra and DSC measurements for $A$ nondeformed, $B$ deformed and $C$ highly deformed polypropylene (PP).

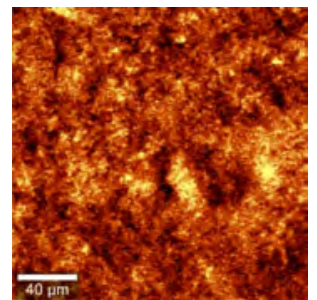

(A) . parallel to MOD, nondeformed

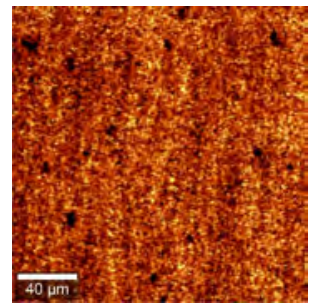

(c) . parallel to MOD, deformed

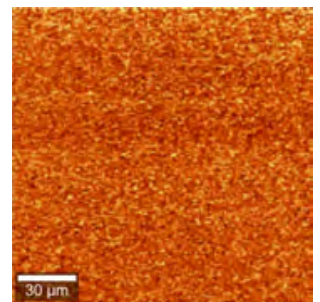

(E) . parallel to MOD, highly deformed

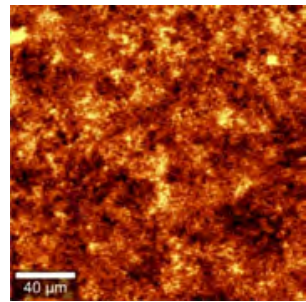

(B) · perpendicular to MOD, nondeformed

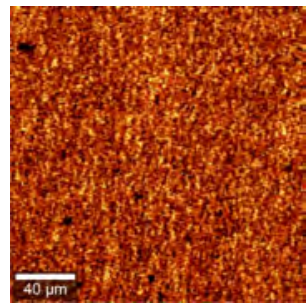

(D) . perpendicular to MOD, deformed

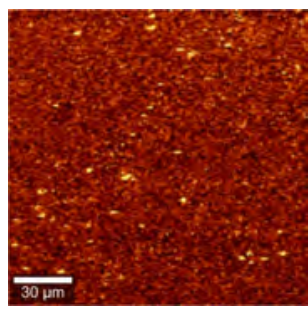

(F) . perpendicular to MOD, highly deformed
FiguRE 6. orientation distribution of PP samples calculated from confocal Raman spectra

Figure 6 shows the orientation distribution of the PP-samples at different MODs and degrees of deformation. The scanned area was $200 \mu m \times 200 \mu m$. It can be seen that the deformation changes the chain orientation from a random distribution to a well-defined periodic arrangement for both laser polarizations. The random distribution of the chains in non-deformed $\mathrm{PP}$ changes to an uniform distribution in highly deformed PP. 


\section{Summary}

With the two selected methods, DSC and Raman spectroscopy, crystallinities were calculated. It was shown that the crystallinity and orientation of the chains increase with the deformation of the polymer. The effect of increasing crystallinity caused by deformation was shown for both polymeric materials, PP and PET.

\section{OutLOOK}

In the current study, PP as one important polymeric material, was analyzed and the results show that confocal Raman microscopy and differential scanning calorimetry are powerful tools for the characterization of the inner structure. Because of the different chemical behavior of different polymers, it is necessary to find useful structure peaks and mathematical formulations for crystallinity and orientation. Finding solutions for other polymer, like PET, will be a subject of future work. In addition, relationships between mechanical properties and the polymer morphology/structure on a micro- and macro-scale are currently under investigation.

\section{ACKNOWLEDGEMENTS}

Robert Führicht for $\mathrm{I}^{\mathrm{A}} \mathrm{T} \mathrm{E}$ X typesetting.

\section{REFERENCES}

[1] J. Martin, M. Ponçot, P. Bourson, et al. Study of the crystalline phase orientation in uniaxially stretched polypropylene by raman spectroscopy: Validation and use of a time-resolved measurement method. Polymer Engineering \& Science 51(8):1607-1616, 2011. DOI:10.1002/pen.21944

[2] M. Fernandez, J. Merino, M. Gobernado-Mitre, J. Pastor. Molecular and lamellar orientation of $\alpha$-and $\beta$-transcrystalline layers in polypropylene composites by polarized confocal micro-raman spectroscopy: Raman imaging by static point illumination. Applied Spectroscopy 54(8):1105-1113, 2000.

[3] J. Rodríguez-Cabello, J. Merino, L. Quintanilla, J. Pastor. Deformation-induced conformational changes in stretched samples of amorphous poly (ethylene terephthalate). Journal of applied polymer science 62(11):1953-1964, 1996.

[4] J. Rodríguez-Cabello, J. Merino, T. Jawhari, J. Pastor. Rheo-optical raman study of chain deformation in uniaxially stretched bulk isotactic polypropylene. Journal of Raman spectroscopy 27(6):463-467, 1996.

[5] A. S. Nielsen, D. Batchelder, R. Pyrz. Estimation of crystallinity of isotactic polypropylene using raman spectroscopy. Polymer 43(9):2671-2676, 2002.

[6] S. Yang, S. Michielsen. Orientation distribution functions obtained via polarized raman spectroscopy of poly (ethylene terephthalate) fibers. Macromolecules 36(17):6484-6492, 2003. DOI:10.1021/ma034486q 